SELECTED PAPER AT NCSP'17

\title{
Path Planning and Traveling Control for Pesticide-Spraying Robot in Greenhouse
}

\author{
Naoki Nakao, Hiroshi Suzuki, Takahiro Kitajima, Akinobu Kuwahara and Takashi Yasuno \\ Graduate School of Advanced Technology and Science, Tokushima University \\ 2-1 Minami-Josanjima, Tokushima 770-8506, Japan \\ E-mail: nakao-n@ee.tokushima-u.ac.jp \\ \{suzuki.hiroshi, yasuno.takashi\} @ tokushima-u.ac.jp
}

\begin{abstract}
This paper describes path-planning and traveling-control algorithms for a pesticide-spraying robot in a greenhouse. In order to search for a suitable path, we applied graph theory and expressed a greenhouse map as a set of nodes and branches. The robot searches for the path from the start node to the goal node through all branches that it needs to spray. Moreover, the robot can identify its position on a map by detecting the shapes of plant beds and walls using a laser range finder (LRF) and can decide which direction to turn. In addition, if its pesticide tank is empty, the robot needs to return to the charge node to obtain more pesticide, and then restarts traveling and spraying. We consider the validity of the path planning and traveling control from simulation results and experimental results obtained using a mock-up model of a greenhouse lane.
\end{abstract}

\section{Introduction}

Agricultural workers in Japan are aging and their number is decreasing. Agricultural work also includes much heavy labor such as making plant beds, harvesting crops and spraying pesticide. Furthermore, it is difficult to employ new workers because of the unstable income. However, it is important to maintain the production of crops. Therefore, it is necessary to produce crops at large-scale farms to produce crops efficiently. However, it is difficult to manage large-scale farms with a few workers. With this background, agricultural automation has attracted attention for cost reduction and ensuring a stable yield.

Recently, several types of agricultural robot have been studied such as harvesting robots, autonomous traveling tractors and pesticide-spraying robots. In particular, the automation of pesticide-spraying in a greenhouses is also important for protecting the health of laborers.

In order for a robot to travel smoothly in a greenhouse, it is necessary to identify its position and for it to travel between plant beds without collision. Generally, a GPS module is used to identify the position outdoors. However, it is difficult to use a GPS module to identify the position of a robot in a greenhouse due to the attenuation and reflection of radio waves by the roof. Therefore, Rafiq et al. proposed an automatic sprayer for a greenhouse that is guided by rail pipes in the greenhouse [1]. However, it is expensive to install guiding objects in a large-scale farm.

We previously developed a pesticide-spraying robot that can travel autonomously in a greenhouse without an additional guiding device. The robot can travel between plant beds without colliding with them and other obstacles [2]. In this paper, we propose a path-planning and self-positioning method using a laser range finder (LRF) so that the pesticidespraying robot can travel around the whole greenhouse. The robot has map data, which is expressed by graph theory, that is used to plan the traveling path before starting to travel. By path planning, the robot decides the direction to turn when it reaches a intersection of lanes. Therefore, the robot can travel around the whole greenhouse. Simulation and experimental results demonstrate the validity of the proposed algorithm.

\section{Pesticide-Spraying Robot}

Figure 1 shows the developed pesticide-spraying robot, which has an LRF and a spraying module. The specifications of the robot are shown in Table 1 . The size of the robot was decided by considering the narrowest lane of the greenhouse. The volume of the pesticide tank is $200 \mathrm{~L}$.

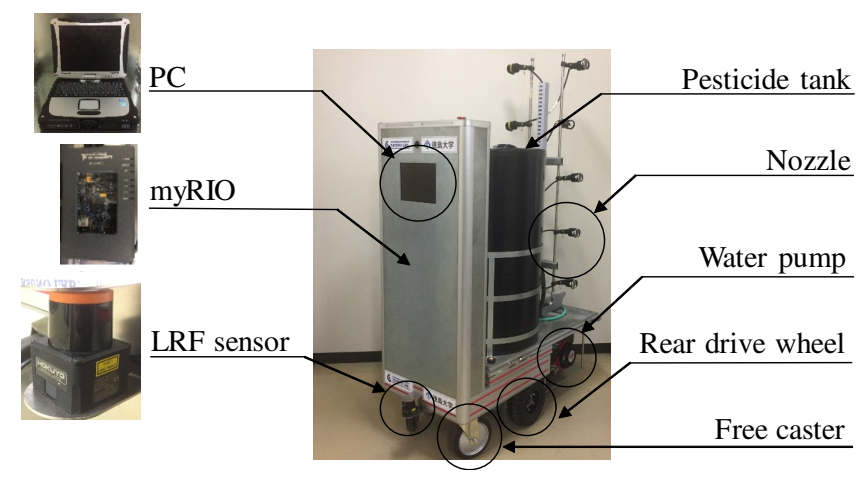

Figure 1: Overview of the pesticide-spraying robot 
The robot is driven by motors with two rear wheels and has a total of four wheels with two front free casters. The reference speed of each motor is calculated from the surrounding information measured by the LRF.

Table 1: Specifications of pesticide-spraying robot

\begin{tabular}{cc}
\hline Size & $540(\mathrm{~W}) \times 1220(\mathrm{~L}) \times 1480(\mathrm{H})$ \\
\hline Pesticide tank & $\phi 557 \times 935 \mathrm{~mm}, 200 \mathrm{~L}$ \\
\hline LRF sensor & Hokuyo UST-10LX \\
\hline Drive motor & Nissei VHLD28L/R-20N400L2 \\
\hline Battery & Lithium ion 24 V, 36 Ah $\times 3($ Parallel $)$ \\
\hline
\end{tabular}

The LRF is mounted in front of the robot and can measure the distance to obstacles in each sensing direction using an infrared laser beam. The specifications of the LRF are shown in Table 2. The robot avoids collisions and detects node positions by measuring obstacles, plant beds, pipes and walls using the LRF.

Table 2: The specifications of the LRF

\begin{tabular}{cc}
\hline Detecting range & $0.06-10 \mathrm{~m}$ \\
\hline Light source & semiconductor $(905 \mathrm{~nm})$ \\
\hline Detection accuracy & $\pm 40 \mathrm{~mm}$ \\
\hline Scanning angle range & $270^{\circ}$ \\
\hline Angular resolution & $0.25^{\circ}$ \\
\hline Scanning time & $25 \mathrm{~ms}$ \\
\hline
\end{tabular}

The spraying module is mounted on the robot. This module is an electrostatic spray type that applies a high voltage to the sprayed pesticide. This ionizes the pesticide particles so that they are attached to the plants efficiently. The spraying module has ten nozzles, which are controlled by an electric signal. Therefore, we can reduce the waste of pesticide by controlling the spraying nozzles and electrostatic spraying. Here, the water pump is driven by an engine because an electric high-pressure pump is too large and heavy to mount on the robot. The control system of the robot was developed using LabVIEW software and the devices are controlled by the I/O device NI myRIO.

\section{Path-Planning Algorithm}

The path-planning algorithm is based on graph theory, which is the study area of graphs in mathematics. A graph is made up of nodes and branches, where the nodes correspond to intersections of lanes in the greenhouse.

Each node has information of the $\mathrm{X}$ and $\mathrm{Y}$ coordinates in the greenhouse, and each branch is expressed by two nodes that it connects. Moreover, branches are classified into two kinds, spray branch which need to be sprayed pesticide, and free branches which do not need spraying. The robot needs to plan a traveling path from the start node to the goal node passing through all the spray branches.

Dijkstra's algorithm is a simple routing algorithm in graph theory. It is useful for finding the shortest path between two nodes. However, it cannot to be directly applied to this problem because the system needs to distinguish between spray branches and free branches. Moreover, the map of the greenhouse is basically a lattice shape, which is a very simple structure in graph theory. Therefore, we proposed a simple pathplanning algorithm to generate a reference path.

Figure 2 shows a flow chart of the proposed path-planning algorithm. Firstly, the system loads the map data and stores the start node as the current node. Then the system adds the node of the nearest spray branch the robot has not yet passed along at the end. It repeats this process until the all spray branches have been included. Here, if the nearest spray branch is not directly connected to the current node, the system inserts relay nodes to connect the nearest branch. The search for the relay nodes is based on depth-first order with priority on $\mathrm{X}$ coordinates. After including the all spray branches, the system adds a goal node with some relay nodes to move the robot to the goal node.

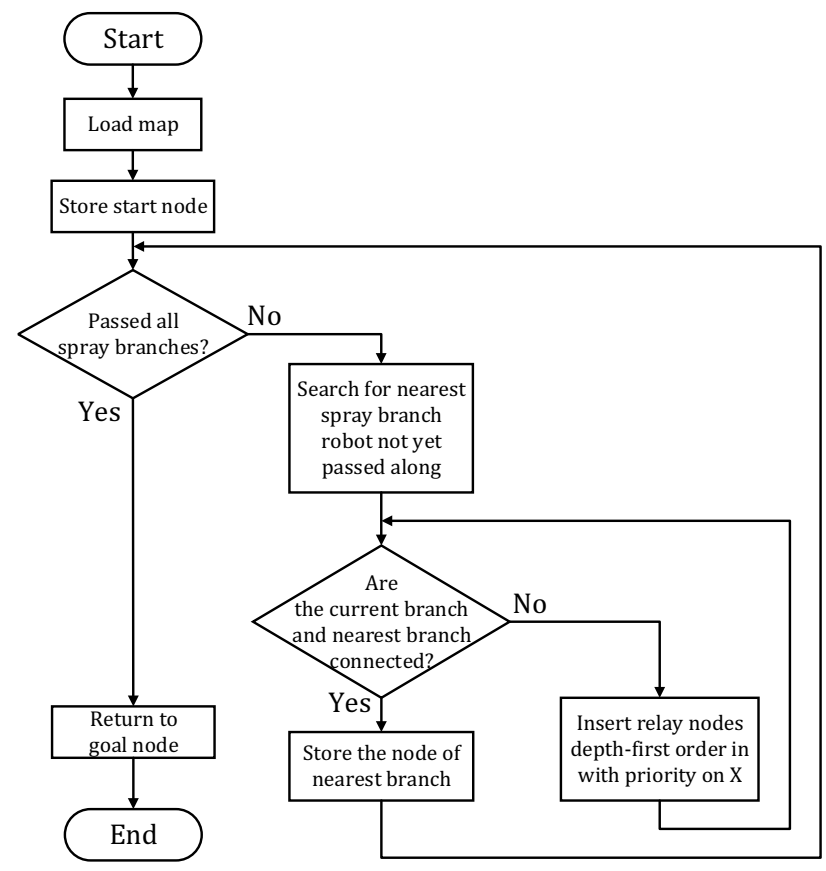

Figure 2: Flow chart of path planning algorithm

\section{Traveling System}

In order to travel along the reference path, it is necessary to identify the robot's position. The robot detects the node position based on surrounding information measured by the LRF. Then, the robot decides the direction to turn when it detects the node position by counting the number of nodes that it has passed. 


\subsection{Node detection}

In this study, we assumed the two cases of node detection shown in Figs. 3 and 4. In these cases, the robot judges that it has reached a node position and updates the node to next one. By updating the node, the robot can travel around the greenhouse along the reference path. Here, we set up the parameters for node detection to correspond to a greenhouse in Anan city, Japan. In the first case shown in Fig.3, the robot is located at the end of a lane and at a corner.

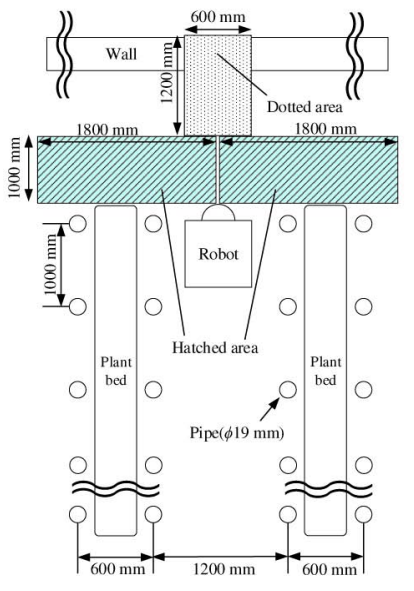

(a) Case of a T-junction

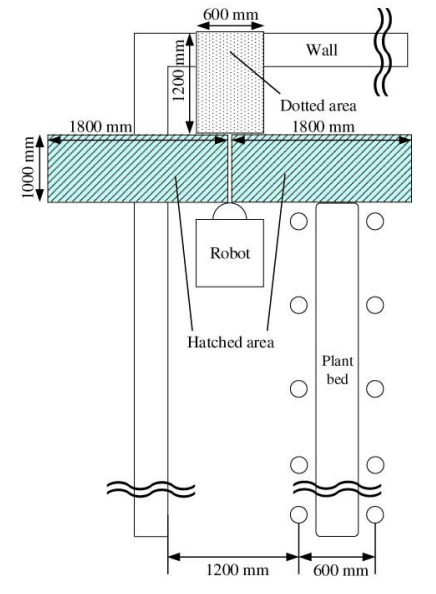

(b) Case of a corner
Figure 3: Case of a wall in front of the robot

The robot detects a position as a node if there is a wall indicated by the dotted area in front and no obstacles either to the left or right, as indicated by the hatched area as shown in Fig.3.

In the second case, the robot is located at a point with a lane to the left or right.

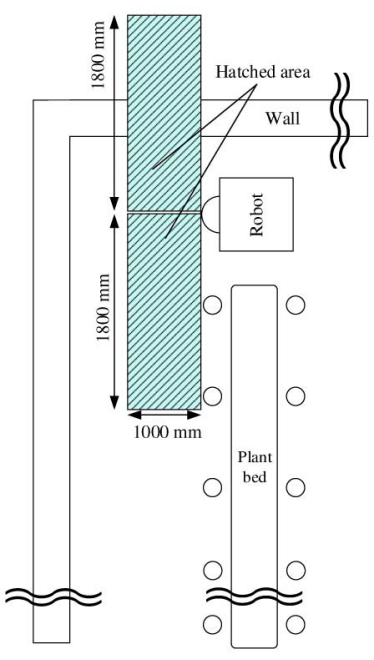

(a) Left

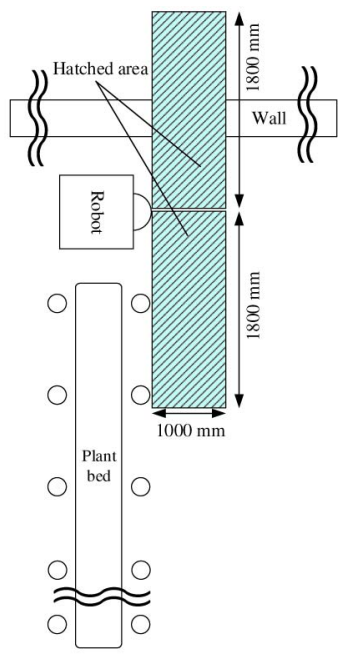

(b) Right
Figure 4: Case of detecting side passage
The robot detects the node if there are no obstacles to the left or right, indicated by the hatched area as shown in Fig.4.

\subsection{Self-position identification}

The odometry method, which is a type of self-position identification method is applied to the robot. In this method, the robot's position is estimated from the travel distance of both wheels. However, it is difficult to estimate the position with sufficient accuracy by only using the odometry method. Therefore, we propose a method combining odometry and node counting. In our proposed method, the robot counts the number of nodes it has passed to recognize the current branch of the reference path. Moreover, the robot adjusts its position estimated by the odometry method to the current node position at the time of node updating. Therefore, the estimated robot's position does not accumulate a measurement error.

\section{Experimental Results}

\subsection{Test environment}

Figures 5(a) and 5(b) respectively show an overview and the dimensions of our test course. In Fig. 5(a), the robot is located at the lower left, which is the start position.

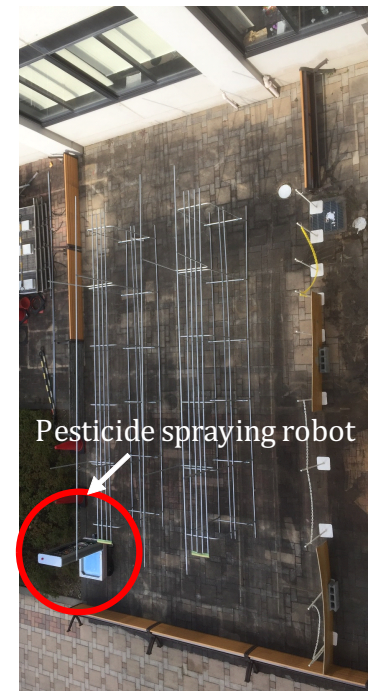

(a) Overview

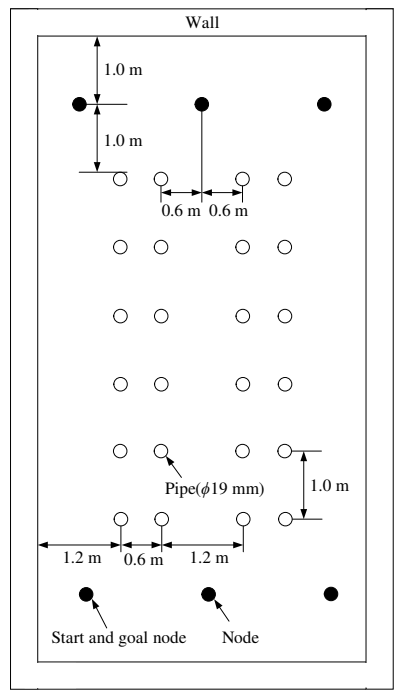

(b) Structure layout included each dimension
Figure 5: Test course for experiment

\subsection{Result of path planning}

Figure 6(a) shows a map of the test course, which is expressed as a non-directed graph. In the figure, the spray branches and free branches are distinguished. Fig.6(b) shows the result of path planning. The robot successfully planned a path that passes along all spray branches from the start node to the goal node. 


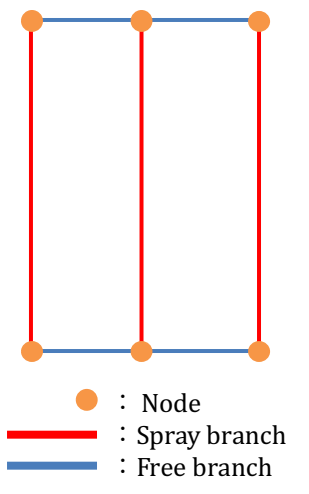

(a) Input data used as the map of the test field

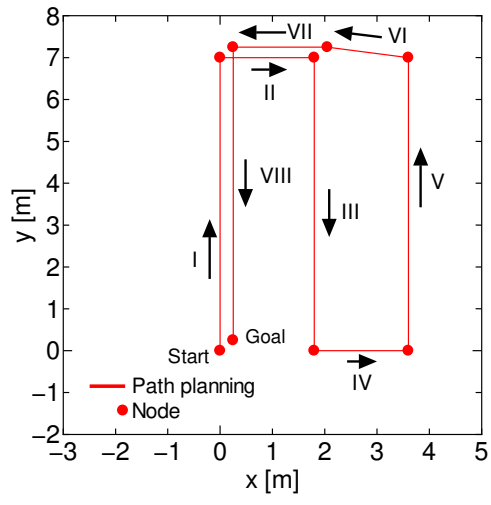

(b) Path planning in the test field
Figure 6: Simulation result of path planning

\subsection{Experimental results of traveling test}

Figure 7 shows the trajectory of the robot as it traveled from the start node to the goal node in the test field estimated by the odometry method. As shown it Fig.7, the odometry method has an error in the angle and distance owing to the sliding of the tire.

On the other hand, Fig. 8 shows the trajectory of the robot after it has been adjusted by referencing the map data when the robot judges that it has reached a node position. It is necessary to know where the robot is currently located so that it can travel along all spray branches. In addition, to travel along all spray branches, the robot must follow the reference path. Therefore, the robot has to adjust the its position traced by the odometry method when it detects that it has reached a node position.

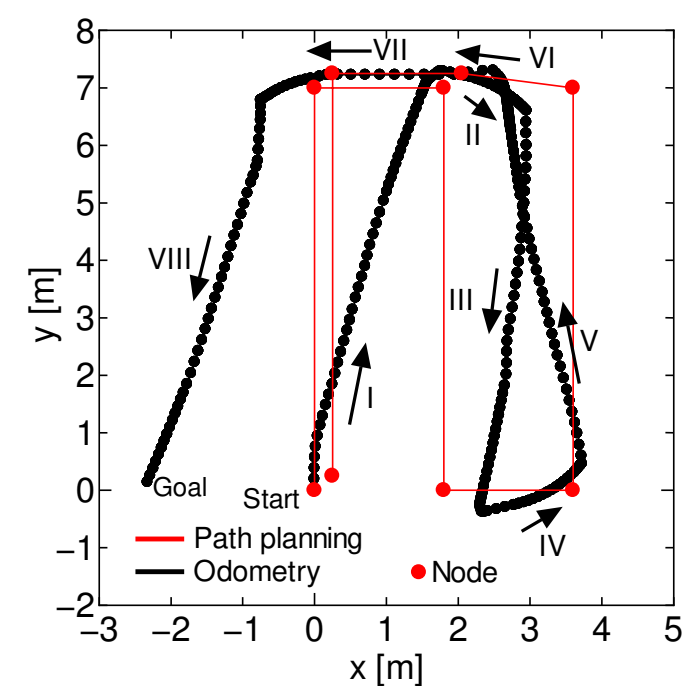

Figure 7: Experimental results obtained only using the odometry method

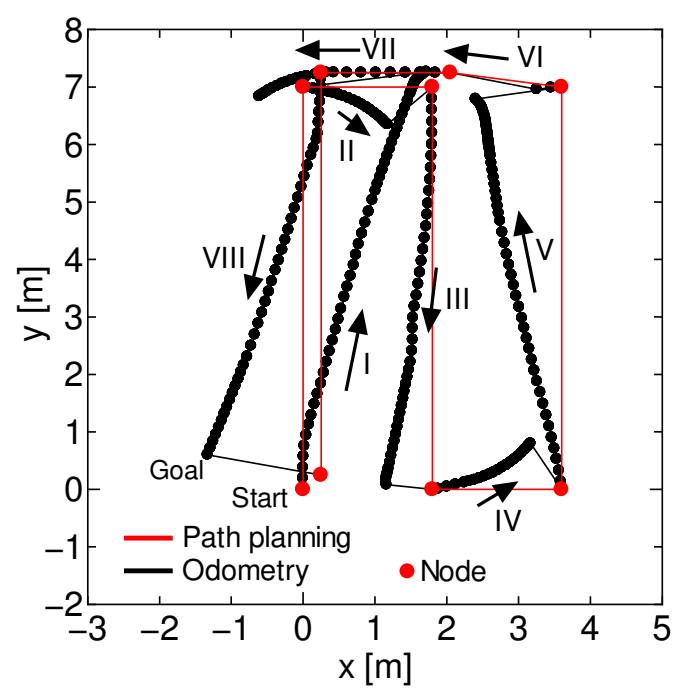

Figure 8: Experimental results when combining the odometry method with the map data

\subsection{Discussion}

The robot can decide the direction to turn and identify its position when it detects that it has reached a node position in order to travel from the start node to the goal node following the reference path. Furthermore, the robot can travel not only in greenhouse but also outside area. If the robot travels outside the area, it can identify its position without any object, such as a GPS module, guiding device, or the magnetic sensor by using these traveling method. In addition, in a different environment, such as a different greenhouse, the robot can travel along all the branches requiring the spraying of a pesticide by simply changing the map.

\section{Conclusions}

In this paper, we proposed path planning as a means of identifying the position of a robot in a greenhouse. Using the proposed method, the robot successfully traveled from the start node to the goal node following the reference path.

As future tasks, we plan to develop a system allowing the robot to distinguish the leaves of crops and other obstacles. This is because crops grow, which may prevent the robot from traveling along a lane between plant beds and from carrying out node detection, depending on the season.

\section{References}

[1] A. Rafiq, D. Kalantari and H. Mashhadimeyghani, Construction and development of an automatic sprayer for greenhouse. Agricultural Engineering International: CIGR Journal, Vol. 16, No. 2, pp. 36-40, 2014.

[2] T. Kitajima, A. Kuwahara, T. Yasuno, T. Fujii, K. Inoue and M. Inoue: Development of autonomous pesticide spray robot and its driving algorithm for greenhouse horticulture, International Design and Concurrent Engineering Conference, No. 52, 2015. 